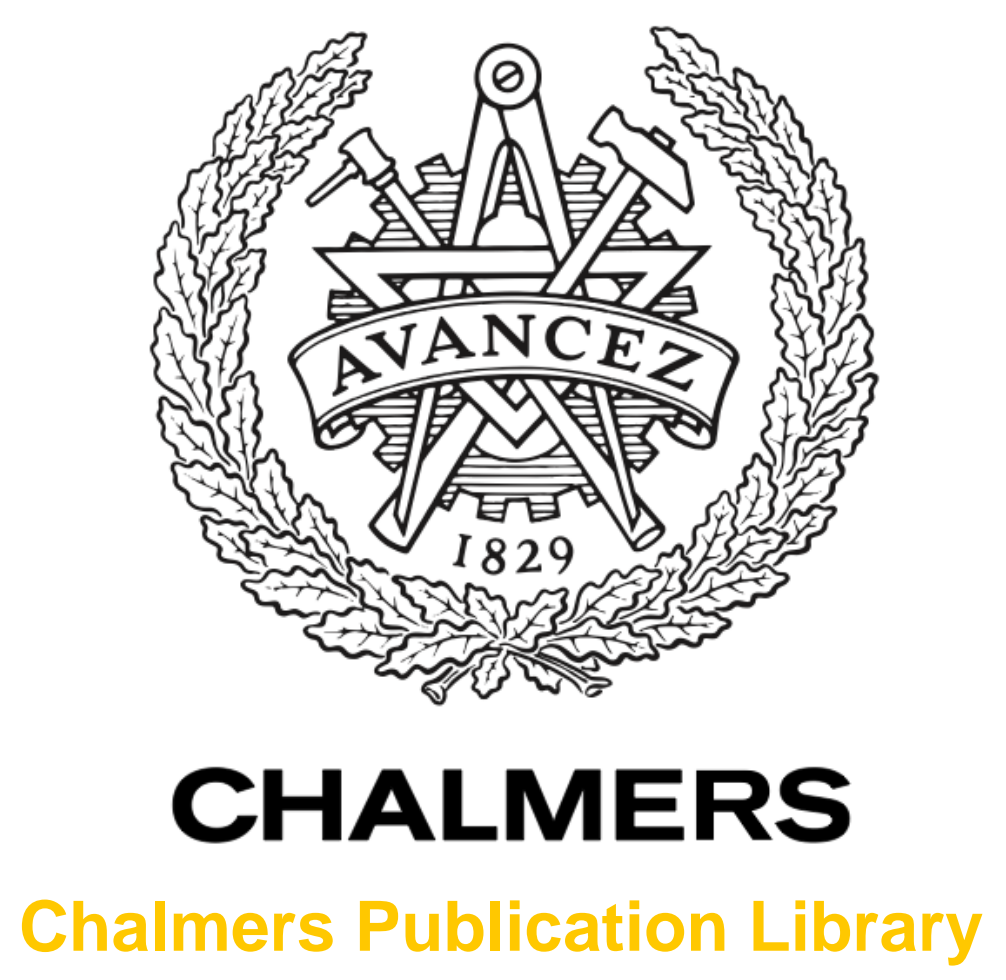

\title{
A simple strategy for mitigating XPM in nonlinear WDM optical systems
}

This document has been downloaded from Chalmers Publication Library (CPL). It is the author's version of a work that was accepted for publication in:

2015 Optical Fiber Communications Conference and Exhibition, OFC 2015; Los Angeles; United States; 22-26 March 2015

Citation for the published paper:

Marsella, D. ; Secondini, M. ; Agrell, E. et al. (2015) "A simple strategy for mitigating XPM in nonlinear WDM optical systems". 2015 Optical Fiber Communications Conference and Exhibition, OFC 2015; Los Angeles; United States; 22-26 March 2015 pp. 7121754.

http://dx.doi.org/10.1364/OFC.2015.Th4D.3

Downloaded from: http://publications.lib.chalmers.se/publication/226000

Notice: Changes introduced as a result of publishing processes such as copy-editing and formatting may not be reflected in this document. For a definitive version of this work, please refer to the published source. Please note that access to the published version might require a subscription. 


\title{
A Simple Strategy for Mitigating XPM in Nonlinear WDM Optical Systems
}

\author{
D. Marsella $^{(1)}$, M. Secondini ${ }^{(1)}$, E. Agrell ${ }^{(2)}$, E. Forestieri $^{(1)}$ \\ (1) TeCIP Institute, Scuola Superiore Sant'Anna, via G. Moruzzi 1, Pisa, Italy \\ (2) Department of Signals and Systems, Chalmers University of Technology, Gothenburg, Sweden \\ d.marsella@sssup.it
}

\begin{abstract}
Resilience to cross-phase modulation (XPM) can be improved by employing multicarrier modulation formats. The impact of the number of subcarriers on the achievable information rate is discussed and a possible XPM compensation strategy is suggested. OCIS codes: $060.2330,060.4510$
\end{abstract}

\section{Introduction}

In wavelength-division multiplexing (WDM) systems with single channel detection, cross-phase modulation (XPM) is known as one of the most important limiting factors to channel capacity [1,2]. Computing the capacity of a nonlinear channel is hardly feasible analytically. However, resorting to the information-theoretic technique of mismatched decoding, lower bounds such as the achievable information rate (AIR) with a given modulation and detection strategy (optimized for an approximated channel) can be evaluated by simulation [3]. The resulting AIR is actually achievable when using this modulation and detection scheme over the real channel. Thus, the AIR (and the tightness of the capacity lower bound) depends on the accuracy of the channel model actually used to design the detector and select the modulation format. Among several models available in the literature [2,4-6], the frequency-resolved logarithmic perturbation (FRLP) model provides an accurate and mathematically tractable description of XPM [2]. According to this model, the observed channel in a WDM system undergoes a time- and frequency-dependent phase rotation $\theta(f ; t)$ due to XPM. The XPM term $\theta(f ; t)$ is a quadratic form of the symbols transmitted on the interfering channels and, in principle, could be exactly evaluated (and compensated for) if the other WDM channels were available. Since this is an unrealistic case, it is more practical performing single channel detection and treating the XPM term as noise. As pointed out in [7], the XPM term $\theta(f ; t)$ exhibits a coherence in time and frequency depending on the optical link characteristics. The coherence time suggests that $\theta(f ; t)$ can be estimated and partly compensated, though using a complex receiver [7]. In this work, by exploiting also the frequency coherence of the XPM term, we propose a simpler approach to design modulation and detection schemes that are more resilient to XPM. We show that multicarrier modulation, typically employed in wireless communications to provide robustness to frequency-selective fading, can also provide robustness to XPM in optical system by reducing the impact of $\theta(f ; t)$ on each subcarrier to that of a "normal" (approximately frequency-independent) phase noise. We consider two different detection strategies for the multicarrier system and investigate the AIR over different fiber-optic links and its dependence on the number of subcarriers.

\section{System model and nonlinearity mitigation}

We consider a WDM system with single-channel detection where each WDM channel employs a multicarrier format. The whole WDM signal propagates through a fiber-optic link according to the nonlinear Schrödinger equation. At the output of the link, the desired channel is selected by a demultiplexing filter and backpropagated to remove intrachannel nonlinearity. As noted in [2], backpropagation does not affect the information rate since it is a reversible operation. Hence, the observed signal is only affected by interchannel nonlinearity, modeled by the XPM term $\theta(f ; t)$, and by the ASE noise accumulated during propagation, modeled as additive white Gaussian noise (AWGN). Since $\theta(f ; t)$ exhibits a coherence in frequency, we approximate it as a piecewise constant function, and in particular as flat over a subcarrier bandwidth. Hence, we describe the cascade of nonlinear fiber-optic link and backpropagation block as $N$ independent parallel subchannels, assuming that each subchannel is affected by additive noise and simple phase noise. Note that, increasing the number of subchannels $N$, while reducing intersymbol interference (ISI) (since $\theta(f ; t)$ tends to become actually independent of frequency on each subchannel bandwidth), worsens inter-subchannel interference (ISCI) (due to the spectral broadening caused by the time-dependence of $\theta(f ; t)$ ). In each subchannel, after matched filtering, the received sample at discrete time $\ell$ is thus approximated as

$$
y_{\ell}=h_{0} x_{\ell} e^{j \theta_{\ell}}+n_{\ell}
$$


(a)

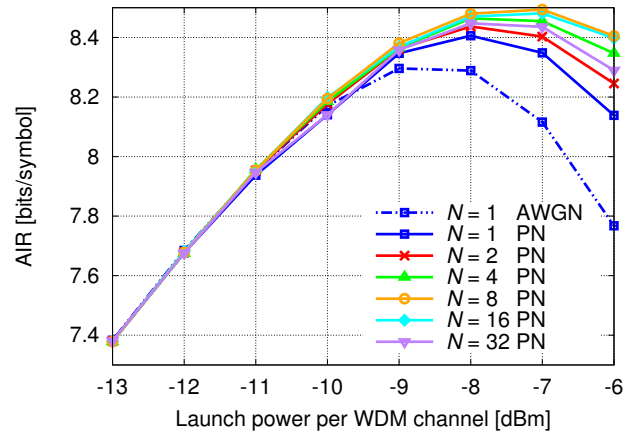

(b)

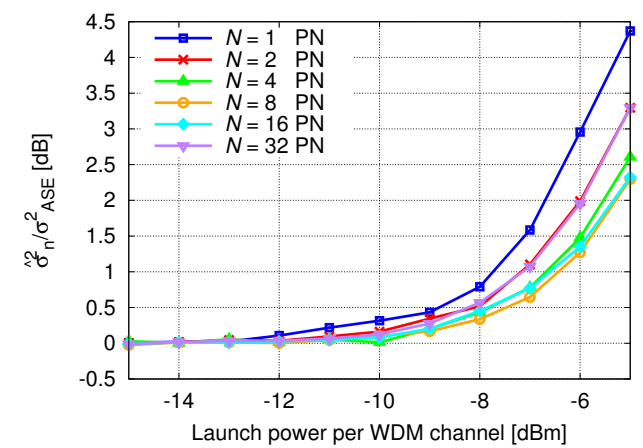

Fig. 1. IDA link, several sub-carriers $N$ : (a) AIR with AWGN or PN detector; (b) additive noise variance.

where $h_{0}$ is a real-valued channel coefficient, $x_{\ell}$ is the transmitted symbol drawn from a given alphabet, $n_{\ell}$ is the additive noise process accounting for ASE noise and ISI/ISCI (as previously discussed), and $\theta_{\ell}$ is the XPM term. We assume that $n_{\ell}$ are i.i.d. zero-mean complex Gaussian variables with variance $\sigma_{n}^{2}, \theta_{\ell}$ are real (possibly correlated) Gaussian variables with mean $\mu_{\theta}$ and variance $\sigma_{\theta}^{2}$, and that $x_{\ell}, n_{\ell}$, and $\theta_{\ell}$ are mutually independent. Given the approximated model (1), we consequently design modulation and detection. Note that we use the simplified channel model (1) to derive suboptimal decoders, which can be implemented with a modest complexity, and not for simulations. The computed AIRs are, according to [3], valid lower bounds on the capacity of the real fiber channel, represented by the NLSE, and achievable by the suboptimal decoders. As shown in [8], the choice of a behavioral model has a strong impact on capacity lower bounds. In our case, we assume that all WDM users transmit with same distribution and power. In particular, we consider i.i.d. Gaussian symbols (capacity-achieving for the AWGN channel) on each subcarrier of each channel. At the receiver side, we consider three different symbol-by-symbol detectors: the AWGN detector, optimized for an AWGN channel; the phase noise (PN) detector, optimized for channel (1) assuming that $\theta_{\ell}$ are uncorrelated; the correlated phase noise (CPN) detector, accounting also for the time correlation of $\theta_{\ell}$ by means of phase noise estimation and compensation before the PN detector $\left(y_{\ell}^{\prime}=y_{\ell} e^{-j \hat{\theta}_{\ell}}\right.$, where $\hat{\theta}_{\ell}$ is estimated as in [7]).

\section{Numerical results}

We consider 3 channels in a single-polarization Nyquist-WDM system ${ }^{1}$ with the same symbol rate $R=1 / T=50 \mathrm{GBd}$ and launch power $P$. In each WDM channel, we employ a multicarrier format with $N$ subcarriers, each with symbol rate $R_{s c}=1 / N T=50 / N G B d$, spectral shape $G_{s c}(f)=\sqrt{N T} \operatorname{rect}(f N T)$, and launch power $P / N$. The simulations are performed by using the split-step Fourier method, considering a $1000 \mathrm{~km}$ dispersion-unmanaged link with attenuation $\alpha=0.2 \mathrm{~dB} / \mathrm{km}$, dispersion $D=17 \mathrm{ps} / \mathrm{nm} / \mathrm{km}$, nonlinear coefficient $\gamma=1.27 \mathrm{~W}^{-1} \mathrm{~km}^{-1}$, and three different amplification schemes: ideal distributed amplification (IDA), 20x50 km lumped amplification, and 10x100 km lumped amplification. At the link output, the central WDM channel is selected by a demultiplexing filter with a lowpass transfer function $\operatorname{rect}(f T)$ and backpropagated. Finally, all subcarriers are selected by a bank of matched filters with equivalent lowpass transfer function $\operatorname{rect}(f N T)$ and sampled at rate $1 / N T$. The system performance is evaluated in terms of AIR, whose estimation at different launch powers is performed in two steps: firstly, a training sequence is used to estimate channel parameters for each subcarrier $\left(h_{0}, \mu_{\theta}, \sigma_{n}^{2}\right.$ for the AWGN detector, plus $\sigma_{\theta}^{2}$ for the PN and CPN detectors) through maximum likelihood estimation; then, the AIR is evaluated through Monte Carlo simulations as in [3].

Fig. 1(a) reports the AIR versus launch power over the IDA link for the AWGN and PN detectors and different numbers of subcarriers $N$. With the AWGN detector, the AIR is independent of $N$ and thus only the single carrier case is shown. As expected, a higher AIR can be achieved with the PN detector. In this case, by increasing $N$, frequency variations of the XPM term $\theta(f ; t)$ over each sub-carrier bandwidth become less relevant, reducing ISI and increasing the AIR up to a limit $(N \simeq 8)$ where the optimum trade-off between ISI and ISCI is reached. After that, spectral broadening of each subcarrier due to the XPM term becomes relevant and the resulting ISCI worsens performance. This behavior is explained by Fig. 1(b), showing the ratio in dB between the estimated variance of the additive noise $\hat{\sigma}_{n}^{2}$ and the variance of the (linearly accumulated) ASE noise $\sigma_{A S E}^{2}$ versus launch power. At low powers, the channel is linear, therefore $\hat{\sigma}_{n}^{2}=\sigma_{A S E}^{2}$ irrespective of the number of subcarriers. On the other hand, increasing the launch power,

\footnotetext{
${ }^{1}$ Preliminary results show that the AIR improvement obtained with the proposed modulation and detection schemes slightly increases when considering more WDM channels, with the same trend shown in [7]. Additional improvements are expected when considering polarizationmultiplexing with cross-polarization mitigation [9].
} 
(a)

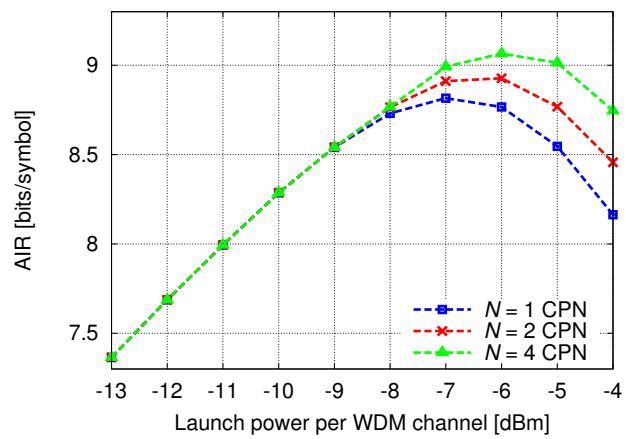

(b)

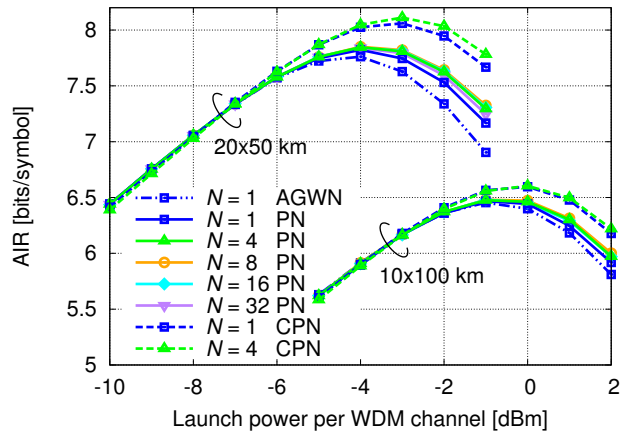

Fig. 2. (a) AIR over the IDA link; (b) AIR over the 10x100 km and 20x50 km links.

$\hat{\sigma}_{n}^{2}$ increases for different causes, depending on $N$ : using a single carrier, $\hat{\sigma}_{n}^{2}$ increases because of the ISI; using 8 subcarriers, it increases due to both ISI and ISCI; and finally, using 32 subcarriers, $\hat{\sigma}_{n}^{2}$ is mainly affected by ISCI. As shown in Fig. 1(b), with 8 subcarriers the additive noise is minimized, leading to the highest AIR. The system performance can be further improved by employing the CPN detector, as shown in Fig. 2(a), with a gain of about 0.6 bits/symbol with respect to the PN detector. Also in this case, the AIR increases by increasing the number of subcarriers. However, as each subcarrier requires a separate tuning of the phase noise estimator, the analysis becomes cumbersome for large $N$ and is performed only up to $N=4$, obtaining a maximum AIR of about 9.1 bits/symbol. This AIR, obtained with a simple symbol-by-symbol detector, equals the AIR obtained in [7] by means of a much more complex detector. Finally, the considered modulation and detection schemes are tested over the 10x100 km and $20 \times 50 \mathrm{~km}$ links, where XPM mitigation is known to be less effective due to a lower time and frequency coherence of the XPM term [7]. As expected, a lower improvement is obtained in these cases: a 4 subcarrier modulation with CPN detection provides an AIR improvement of about 0.4 and $0.2 \mathrm{bit} / \mathrm{symbol}$ over the $20 \times 50$ and $10 \times 100 \mathrm{~km}$ link, respectively, compared to single carrier modulation with AWGN detection.

\section{Conclusions}

The achievable rate, which is a lower bound on channel capacity, can be improved by using a multicarrier modulation format and a simple XPM compensation strategy. The technique outlined here can achieve the same results obtainable by more complex detection strategies as those in [7].

\section{Acknowledgment}

This work was supported in part by the Italian MIUR under the FIRB project COTONE.

\section{References}

[1] A. Mecozzi and R.-J. Essiambre, "Nonlinear Shannon limit in pseudolinear coherent systems," J. Lightwave Technol. 30, 2011-2024 (2012).

[2] M. Secondini, E. Forestieri, and G. Prati, "Achievable information rate in nonlinear WDM fiber-optic systems with arbitrary modulation formats and dispersion maps," J. Lightwave Technol. 31, 3839-3852 (2013).

[3] D. Arnold, H.-A. Loeliger, P. Vontobel, A. Kavcic, and W. Zeng, "Simulation-based computation of information rates for channels with memory," Information Theory, IEEE Transactions on 52, 3498-3508 (2006).

[4] P. Poggiolini, "The GN model of non-linear propagation in uncompensated coherent optical systems," J. Lightwave Technol. 30 , 3857-3879 (2012).

[5] R. Dar, M. Feder, A. Mecozzi, and M. Shtaif, "Time varying ISI model for nonlinear interference noise," in "Opt. Fiber Commun. Conf.", (Optical Society of America, 2014), p. W2A.62.

[6] E. Agrell, A. Alvarado, G. Durisi, and M. Karlsson, "Capacity of a nonlinear optical channel with finite memory," J. of Lightwave Technol. 32, 2862-2876 (2014).

[7] M. Secondini and E. Forestieri, "On XPM mitigation in WDM fiber-optic systems," IEEE Photon. Technol. Lett. DOI: 10.1109/LPT.2014.2353217.

[8] E. Agrell and M. Karlsson, "WDM channel capacity and its dependence on multichannel adaptation models," in "Opt. Fiber Commun. Conf.", (Optical Society of America, 2013), p. OTu3B.4.

[9] R. Dar, O. Geller, M. Feder, A. Mecozzi, and M. Shtaif, "Mitigation of inter-channel nonlinear interference in WDM systems," in "Europ. Conf. on Opt. Commun. (ECOC)," (2014). 